\title{
Restoring ancient text using deep learning: a case study on Greek epigraphy
}

\author{
Yannis Assael $^{1,2, \dagger}$, Thea Sommerschield ${ }^{1, \dagger}, \quad$ Jonathan Prag $^{1}$ \\ University of Oxford $^{1}$, DeepMind ${ }^{2}$
}

\begin{abstract}
Ancient History relies on disciplines such as Epigraphy, the study of ancient inscribed texts, for evidence of the recorded past. However, these texts, "inscriptions", are often damaged over the centuries, and illegible parts of the text must be restored by specialists, known as epigraphists. This work presents PYTHIA, the first ancient text restoration model that recovers missing characters from a damaged text input using deep neural networks. Its architecture is carefully designed to handle longterm context information, and deal efficiently with missing or corrupted character and word representations. To train it, we wrote a nontrivial pipeline to convert PHI, the largest digital corpus of ancient Greek inscriptions, to machine actionable text, which we call PHI-ML. On PHI-ML, PythiA's predictions achieve a $30.1 \%$ character error rate, compared to the $57.3 \%$ of human epigraphists. Moreover, in $73.5 \%$ of cases the ground-truth sequence was among the Top-20 hypotheses of PYTHIA, which effectively demonstrates the impact of this assistive method on the field of digital epigraphy, and sets the state-of-the-art in ancient text restoration.
\end{abstract}

\section{Introduction}

One of the key sources for Ancient History is the discipline of epigraphy, which delivers firsthand evidence for the thought, society and history of ancient civilisations. Epigraphy is the study of documents, "inscriptions", written on a durable surface (stone, ceramic, metal) by individuals, groups and institutions of the past (Davies and Wilkes, 2012). Only a small minority of surviving inscriptions are fully legible and complete, as many have been damaged in time (Figure 1). An epigraphist must then hypothesise how much text is missing,

\footnotetext{
${ }^{\dagger}$ These authors contributed equally to this work.
}

and what it might have originally been. These hypotheses are called "restorations" (Bodel, 2012). The present work offers a fully automated aid to the epigraphist's restoration task.

Restoring text is a complex and time-consuming task (Woodhead, 1967; Mattingly, 1996). Epigraphists rely on accessing vast repositories of information to find textual and contextual "parallels" (recurring expressions in similar documents). These repositories primarily consist in a researcher's mnemonic repertoire of such parallels, and in digital corpora for performing "string matching" searches (The Packard Humanities Institute, 2005; Clauss, 2012). However, minor differences in the search query can exclude or obfuscate relevant results, making it hard to estimate the true probability distribution of possible restorations. To the best of our knowledge, this is the first work to bypass the constraints of current epigraphic methods by means of a fully automated deep learning model, PyтнiA, which aids the task of ancient text restoration. It is supplemented by PHI-ML, an epigraphic dataset of a machine actionable text. PythiA takes as input a sequence of damaged text, and is

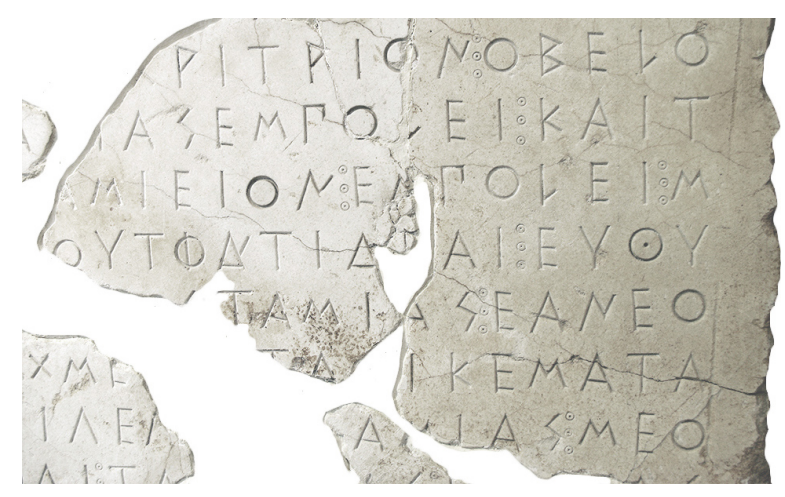

Figure 1: Damaged inscription: a decree concerning the Acropolis of Athens (485/4 BCE). IG $\mathrm{I}^{3} 4 \mathrm{~B}$. (CC BY-SA 3.0, WikiMedia) 
trained to predict character sequences comprising the hypothesised restorations. It works both at a character- and a word-level, thereby effectively handling incomplete or missing words. PYTHIA can furthermore be used by all disciplines dealing with ancient texts (philology, papyrology, codicology) and applies to any language (ancient or modern). To aid and encourage future research, PYTHIA and PHI-ML have been open-sourced at http://tiny.cc/ancient-text-restoration.

\section{Related work}

Natural language processing (NLP) has dealt with tasks akin to text restoration. Indeed, standard count-based n-gram language models (LM) share with epigraphists the "parallel-finding" approach. $\mathrm{N}$-gram models are outperformed by neural language models, which operate at a word-level (Mikolov et al., 2010, 2011), at a subword- or character-level (Sutskever et al., 2011; Mikolov et al., 2012; Botha and Blunsom, 2014), or a combination of both, known as character-aware language models (Miyamoto and Cho, 2016; Kim et al., 2016; Hwang and Sung, 2017). Despite our efforts to include BERT (Devlin et al., 2018) in our evaluation, we found that the excessive resources required did not allow for training on a single GPU. Text restoration also shares similarities with machine reading comprehension (Hermann et al., 2015; Kočiskỳ et al., 2018), and cloze deletion tests (Hill et al., 2016; Bajgar et al., 2017; Fedus et al., 2018; Xie et al., 2018; Zhang et al., 2018). Although word-level language modelling is capable of capturing context information more efficiently than character-level alternatives, damaged inscriptions preserve only limited parts of words, complicating the learning of representations. To overcome this issue, PYTHIA works simultaneously at both a character- and a wordlevel, thereby capturing long-term dependencies ("context information").

Finally, several works have used machine learning to study ancient inscriptions, focusing on assistive tools (Roued-Cunliffe, 2010), optical character recognition and visual analysis (Terras and Robertson, 2006; Garz et al., 2014; Soumya and Kumar, 2014; Shaus, 2017; Hussien et al., 2015; Amato et al., 2016; Can et al., 2016; Suganya and Murugavalli, 2017; Palaniappan and Adhikari, 2017; Avadesh and Goyal, 2018), writer identification (Tracy and Papaodysseus, 2009; Panagopou- los et al., 2009; Faigenbaum-Golovin et al., 2016), text analysis (Rao et al., 2009b,a, 2010; Yadav et al., 2010; Lee and Haug, 2010; Vatri and McGillivray, 2018), and machine translation (Pagé-Perron et al., 2017; Luo et al., 2019).

\section{Generating PHI-ML}

Due to availability of digitised epigraphic corpora, PyTHIA has been trained on ancient Greek (henceforth, "AG") inscriptions, written in the ancient Greek language between $7^{\text {th }}$ century BCE and $5^{\text {th }}$ century CE. We chose AG epigraphy as a case study for two reasons: a) the variability of contents and context of the AG epigraphic record makes it an excellent challenge for NLP; b) several digital AG textual corpora have been recently created, the largest ones being PHI (The Packard Humanities Institute, 2005; Gawlinski, 2017) for epigraphy; Perseus (Smith et al., 2000) and First1KGreek (Crane et al., 2014) for ancient literary texts.

When restoring damaged AG inscriptions, the epigraphists' conjectures on the total number of missing characters are guided by grammatical and syntactical considerations, as well as by the reconstructed graphical layout of the inscription. Conjectured missing characters are conventionally marked with hyphens, one hyphen equating to one missing character. Additionally, epigraphists traditionally convert edited texts to lower case and add punctuation and diacritics, which are generally absent from the original inscription. These conventions were also used in PHI.

Because human annotations in PHI were noisy and often syntactically inconsistent (Iversen, 2007), we wrote a pipeline to convert it into a machine actionable text. We first computed the character frequencies and standardised the AG alphabet to include all core characters, including all accentuation (147 characters), numbers, spaces and punctuation marks. Two additional characters were introduced: '-' representing a missing character, and '?' signifying a character to be predicted. Then we wrote regular expressions to replace all AG numerical notations appearing in the texts with 0 to avoid numerical correlations, strip the remaining punctuation marks, remove the conventional epigraphical symbols surrounding certain characters ("Leiden Conventions"), and discard notes whose content was not in Greek. We then proceeded to clear human comments, fix the spacing and cases of duplicate punctuation, 
and filtered the resulting text so as to retain only the restricted alphabetical characters. The texts with fewer than 100 characters were also discarded. Lastly, we matched the number of missing characters with those conjectured by epigraphists, thereby converting the length value to an equal number of '-' symbols.

The resulting dataset is named PHI-ML, and consists of more than 3.2 million words (Table 1). The inscriptions whose PHI IDs ended in $\{3,4\}$ (every inscription in PHI was assigned a unique identifier when the original corpus was created) were held out and used respectively as test and validation sets.

\begin{tabular}{|r|r|r|r|}
\hline Split & Inscriptions & Words & Chars \\
\hline Train & 34,952 & $2,792 \mathrm{k}$ & $16,300 \mathrm{k}$ \\
Valid & 2,826 & $211 \mathrm{k}$ & $1,230 \mathrm{k}$ \\
Test & 2,949 & $223 \mathrm{k}$ & $1,298 \mathrm{k}$ \\
\hline
\end{tabular}

Table 1: Statistics for the PHI-ML corpus.

\section{Restoring text using Pythia}

Pythia's architecture is a sequence-tosequence (Sutskever et al., 2014) based neural network architecture, consisting of a Long-Short Term Memory (LSTM) (Hochreiter and Schmidhuber, 1997) encoder, an LSTM decoder, and an attention mechanism introduced by Luong et al. (2015); Bahdanau et al. (2014). The encoder takes an inscription text $x$ as input, where the symbol '-' denotes the missing characters, and '?' the blanks to be predicted. The input characters are first passed through a lookup table with learnable embedding vectors. Next, the encoded sequence is used as input for the decoder, which is trained to predict the content of the '?' characters, as shown in Figure 2. Attention allows the decoder to "attend" to parts of the input sequence relevant to the current output, thus improving the modelling of long-term dependencies. To further improve performance, we designed PYTHIA's encoder to take an additional input stream of word embeddings, as it is difficult to model the word-level context using only character-level information. Thus, we generated a list of the 100k most frequent words appearing in PHI-ML, and using a separate lookup table we concatenated at each time-step the embedding of each character with the embedding of the word it belongs to. Words that do not appear in the list, or that contain missing characters were mapped to 'unk', an embedding for unknown words. Figure 2 illustrates PYTHIA processing the phrase unóćv ar rav. Finally, to allow better modelling we used a bidirectional LSTM encoder and refer to this architecture as PYTHIA-BI-WORD. Further details are given in Appendix A.

Obtaining suggestions. To better aid the epigraphist's task, PYTHIA returns multiple predictions as well as the level of confidence for each result, rather than a single prediction per text restoration. Specifically, we provide a set of the Top 20 predictions decoded using beam search.

\section{Experimental evaluation}

The ground-truths for incomplete epigraphic texts were lost over millennia. Consequently, in order to generate a ground-truth sequence, we artificially removed part of the input text and treated this as the ground-truth sequence. On each training step we selected an inscription and sampled a start index and a length value $\in[100,1000]$, and extracted the context text $x$, which was then used as input. Within $x$, we sampled a new start index and length $\in[1,10]$ to select the target sequence $y$; its characters' positions were replaced with the special symbol '?', which denotes the positions to be predicted. The test and validation sets used the maximum context length. Beam search with a beam width of 100 was used to decode hypothesis. To simplify comparisons, all AG accentuation was discarded, as inputting accents was timeconsuming for the human evaluations described in the following paragraph. This decision did not noticeably influence the reported scores.

\subsection{Methods evaluated}

AnCiEnT historian. Because text restoration is an extremely time-consuming task even for an expert epigraphist, we set out to evaluate the difficulty of the restoration task at hand - and thereby judge the impact of our work - with the help of two doctoral students with epigraphical expertise. The scholars were allowed to use the training set to search for "parallels", and made an average of 50 restorations in 2 hours, with a $57.3 \%$ character error rate (CER).

LM PHILOLOGY. To evaluate the performance of a model using "parallels", we trained a LM. Since large parts of the text are garbled, making complete words unidentifiable, and because BERT was not an option, the LM works at a character- 

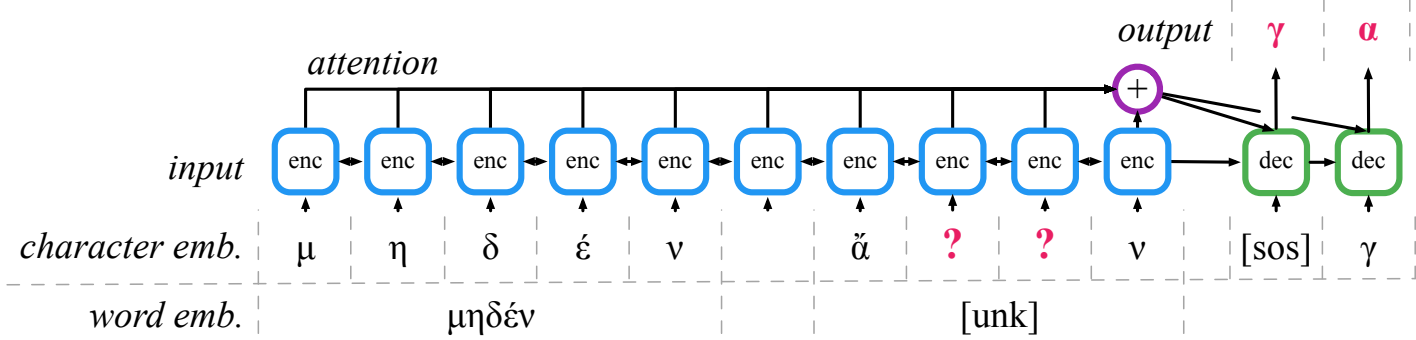

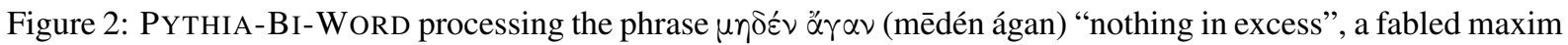
inscribed on Apollo's temple in Delphi. The letters " $\gamma \alpha$ " are the characters to be predicted, and are annotated with '?'. Since $\alpha ? ? \nu$ is not a complete word, its embedding is treated as unknown ('unk'). The decoder outputs correctly " $\gamma \alpha$ ".

level and is based on the setup of Zaremba et al. (2015) (Appendix B). The LM was trained on two larger digital corpora of literary AG texts ("philology"), First1KGreek and Perseus, and evaluated on PHI-ML.

LM PHILOLOGY \& EPIGRAPHY. LM jointly trained on First1KGreek, Perseus and PHI-ML.

LM EPIGRAPHY. LM trained on PHI-ML.

PYTHIA-UNI. An ablation architecture, using only characters as input and unidirectional LSTM.

Pyтhia-BI. Similar to the PYThiA-Uni ablation, but with a bidirectional LSTM.

PYTHIA-BI-WORD. This is our proposed model of choice, which uses a bidirectional LSTM and both characters and words as inputs.

\subsection{Results}

The aforementioned methods were evaluated using: a) the character error rate (CER) of the top prediction and the target sequence, b) the Top20 accuracy score, where we ascertain whether the ground-truth sequence exists within the first 20 predictions. The latter evaluates the effectiveness of PYTHIA as an assistive tool providing restoration suggestions to epigraphists. As shown in Table 2, the ancient historians' restorations had a CER of $57.3 \%$, which is telling of the difficulty of the task. The language model trained on epigraphic datasets performed slightly better, with a CER of $57.3 \%$. Interestingly, the two attempts to use larger philological datasets performed worse. This is very likely due to a divergence in epigraphical and literary cultures. The CER of the unidirectional PYTHIA-UNI and the bidirectional PYTHIA-BI alternatives were $42.2 \%$ and $32.5 \%$ respectively. The top score was therefore achieved by the bidirectional PYTHIA-BIWORD, which took both word and character embeddings as inputs, with a CER of $30.1 \%$. Fur-

\begin{tabular}{|l|r|r|}
\hline Method & CER & Top-20 \\
\hline Ancient Historian & $57.3 \%$ & - \\
\hline LM Philology & $68.1 \%$ & $26.0 \%$ \\
LM Philology \& Epigraphy & $65.0 \%$ & $28.8 \%$ \\
LM Epigraphy & $52.7 \%$ & $47.0 \%$ \\
\hline PYTHIA-UNI & $42.2 \%$ & $60.6 \%$ \\
PYTHIA-BI & $32.5 \%$ & $71.1 \%$ \\
PYTHIA-BI-WORD & $\mathbf{3 0 . 1} \%$ & $\mathbf{7 3 . 5} \%$ \\
\hline
\end{tabular}

Table 2: Predictive performance on PHI-ML.

thermore, the ground-truth appeared among the 20 most probable predictions of PYTHIA-BI-WORD $73.5 \%$ of the times, which indicates that it could be a uniquely effective assistive tool.

\subsection{The importance of context}

The presence of context information is a determining factor in the accuracy of epigraphic restorations. We therefore evaluated the impact of different textual lengths acting as augmented context on the Top-20 accuracy measure of PYTHIA. As can be seen in Figure 3, the correlation between the "context length" and the predictive performance of our model is positive. Specifically, the performance peaks around 500 characters of context

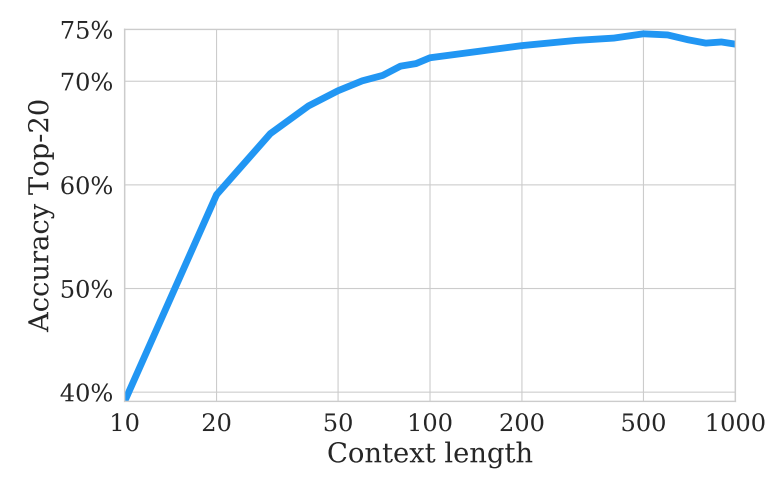

Figure 3: Predictive performance of the PYTHIA-BIWORD model under different context lengths. 
length. Furthermore, Figure 3 exemplifies the increased difficulty faced by the model when only a short context length (e.g. 20 characters) is offered. The latter scenario recalls the similar difficulties encountered by string-matching and "parallel" search approaches, where the search queries would often be short.

\subsection{Visualising PythiA's attention}

We set up an example modifying lines b. 8 - c.5 of the inscription MDAI(A) 32 (1907) 428, 275 (PHI ID PH316753), to evaluate PYTHIA's receptiveness to context information and visualise the attention weights at each decoding step. In the text of Figure 4, the last word is a Greek personal

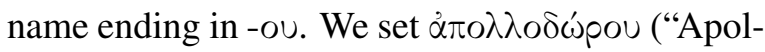
lodorou") as the personal name, and hid its first 9 characters. This name was specifically chosen because it already appears within the input text. Figure 4 illustrates the attention weights for decoding the first 4 missing characters. To aid visualisation, the weights were separately scaled between 0 and 1 within the area of the characters to be predicted ('?') in green, and of the rest of the text in blue; the magnitude is represented by the colour intensity. As can be seen, PYTHIA is attending to the contextually-relevant parts of

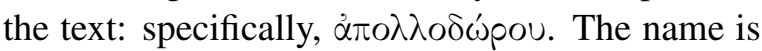
correctly predicted. As a litmus test, we substi-

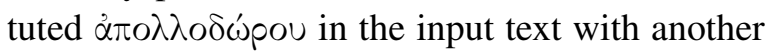

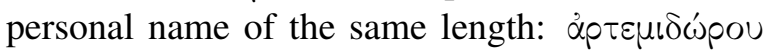
("Artemidorou"). The predicted sequence alters

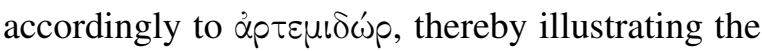
importance of context in the prediction process.

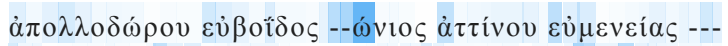

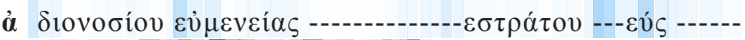

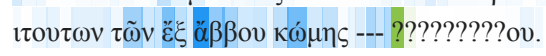

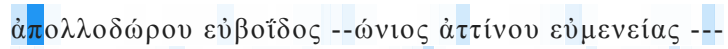

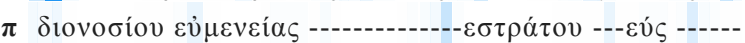

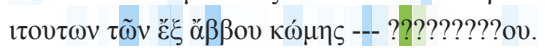

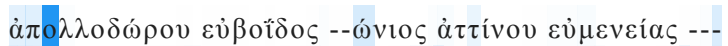

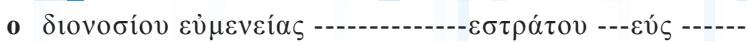

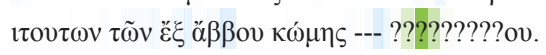

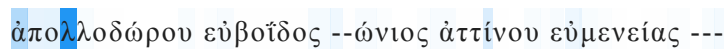

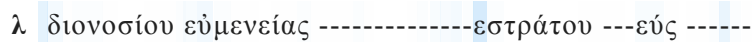

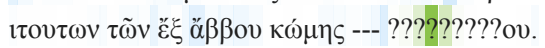

Figure 4: Visualisation of the attention weights for the decoding of the first 4 missing characters. The groundtruth text $y=\alpha \pi \sigma \lambda \lambda o \delta \omega \rho \rho$ appears in the input text, and PYTHIA attends to the relevant parts of the sequence.

\subsection{Restoring full texts}

We then applied PythiA iteratively in order to predict all the missing text of an AG inscription, comparing PYTHIA's predictions with an edition of reference (Rhodes and Osborne, 2003). In Figure 5 the correct restorations are highlighted in colour blue and erroneous ones are in purple. In a real-world scenario, PYTHIA would provide more than one hypothesis to the epigraphist. The ground-truth sequence did in fact exist within the Top-20 hypotheses in nearly all cases, illustrating the efficacy of such technologies when paired with human decision-making.

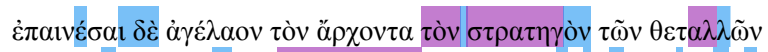

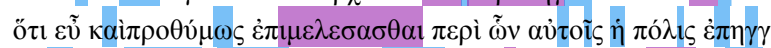

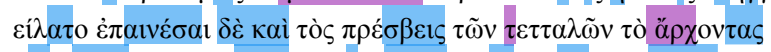

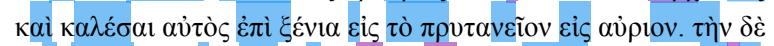

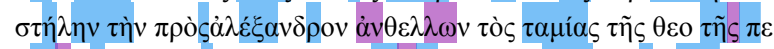

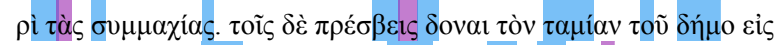

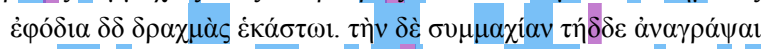

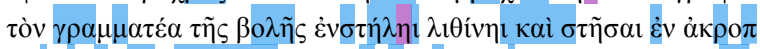

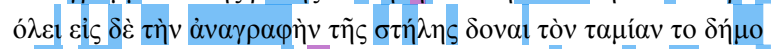

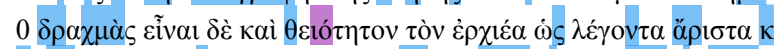

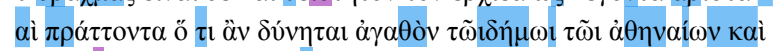

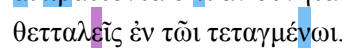

Figure 5: Sample restoration of the inscription $I G \mathrm{II}^{2}$ 116, lines $34-48$. Restorations are in colour blue when correct, purple when incorrect.

\section{Conclusions}

PythiA is the first ancient text restoration model of its kind. Our experimental evaluation and ablation studies illustrate the validity of our design decisions, and illuminate the ways PYTHIA can assist, guide and advance the ancient historian's task - and digital humanities proper. The combination of machine learning and epigraphy has the potential to impact meaningfully the study of inscribed textual cultures, both ancient and modern. By open-sourcing PYTHIA, and PHI-ML's processing pipeline, we hope to aid future research and inspire further interdisciplinary work.

\section{Acknowledgements}

We would like to thank Leah Lazar for her valuable contributions; Brendan Shillingford, Misha Denil, Çağlar Gülçehre, and Nando de Freitas for the helpful comments and discussions; and the Packard Humanities Institute for having made this data digitally available. 


\section{References}

Giuseppe Amato, Fabrizio Falchi, and Lucia Vadicamo. 2016. Visual recognition of ancient inscriptions using convolutional neural network and fisher vector. Journal on Computing and Cultural Heritage, 9(4):21.

Meduri Avadesh and Navneet Goyal. 2018. Optical character recognition for sanskrit using convolution neural networks. In International Workshop on Document Analysis Systems, pages 447-452.

Dzmitry Bahdanau, Kyunghyun Cho, and Yoshua Bengio. 2014. Neural machine translation by jointly learning to align and translate. In International Conference on Learning Representations.

Ondrej Bajgar, Rudolf Kadlec, and Jan Kleindienst. 2017. Embracing data abundance: Booktest dataset for reading comprehension. In International Conference on Learning Representations Workshop.

Samy Bengio, Oriol Vinyals, Navdeep Jaitly, and Noam Shazeer. 2015. Scheduled sampling for sequence prediction with recurrent neural networks. In Advances in Neural Information Processing Systems, pages 1171-1179.

John Bodel. 2012. Epigraphy and the ancient historian. In Epigraphic Evidence, pages 27-82. Routledge.

Jan Botha and Phil Blunsom. 2014. Compositional morphology for word representations and language modelling. In International Conference on Machine Learning, pages 1899-1907.

Gülcan Can, Jean-Marc Odobez, and Daniel GaticaPerez. 2016. Evaluating shape representations for maya glyph classification. Journal on Computing and Cultural Heritage, 9(3):14.

Manfred Clauss. 2012. Epigraphik-datenbank claussslaby. Accessed on 2019-04-24.

Gregory Ralph Crane, Monica Berti, Annette Geßner, Matthew Munson, and Tabea Selle. 2014. Open greek and latin project. http://www.dh.uni-leipzig. de/wo/projects/open-greek-and-latin-project/. Accessed on 2019-04-24.

John Davies and John Wilkes. 2012. Epigraphy and the historical sciences. British Academy.

Jacob Devlin, Ming-Wei Chang, Kenton Lee, and Kristina Toutanova. 2018. Bert: Pre-training of deep bidirectional transformers for language understanding. arXiv preprint arXiv: 1810.04805 .

Shira Faigenbaum-Golovin, Arie Shaus, Barak Sober, David Levin, Nadav Na'aman, Benjamin Sass, Eli Turkel, Eli Piasetzky, and Israel Finkelstein. 2016. Algorithmic handwriting analysis of judaha's military correspondence sheds light on composition of biblical texts. National Academy of Sciences, 113(17):4664-4669.
William Fedus, Ian J. Goodfellow, and Andrew M. Dai. 2018. Maskgan: Better text generation via filling in the __. In International Conference on Learning Representations.

Angelika Garz, Nicole Eichenberger, Marcus Liwicki, and Rolf Ingold. 2014. Hisdoc 2.0: Toward computer-assisted paleography. Manuscript Cultures, Natural Sciences and Technology in Manuscript Studies, 7.

Laura Gawlinski. 2017. Review: Packard humanities institute's searchable greek inscriptions. https://classicalstudies.org/scs-blog/lauragawlinski/review-packard-humanities-institutessearchable-greek-inscriptions. Accessed on 2019-08-26.

Karl Moritz Hermann, Tomas Kocisky, Edward Grefenstette, Lasse Espeholt, Will Kay, Mustafa Suleyman, and Phil Blunsom. 2015. Teaching machines to read and comprehend. In Advances in neural information processing systems, pages 16931701.

Felix Hill, Antoine Bordes, Sumit Chopra, and Jason Weston. 2016. The goldilocks principle: Reading children's books with explicit memory representations. In International Conference on Learning Representations.

Sepp Hochreiter and Jürgen Schmidhuber. 1997. Long short-term memory. Neural computation, 9(8):1735-1780.

Rana S Hussien, Azza A Elkhidir, and Mohamed G Elnourani. 2015. Optical character recognition of arabic handwritten characters using neural network. In International Conference on Computing, Control, Networking, Electronics and Embedded Systems Engineering, pages 456-461. IEEE.

Kyuyeon Hwang and Wonyong Sung. 2017. Characterlevel language modeling with hierarchical recurrent neural networks. In International Conference on Acoustics, Speech and Signal Processing, pages 5720-5724. IEEE.

Paul A. Iversen. 2007. The Packard Humanities Institute - Greek epigraphy project and the revolution in Greek epigraphy. Abgadiyat, 2.1.

Yoon Kim, Yacine Jernite, David Sontag, and Alexander M Rush. 2016. Character-aware neural language models. In American Association for Artificial Intelligence.

Diederik P. Kingma and Jimmy Ba. 2015. Adam: A method for stochastic optimization. In International Conference on Learning Representations.

Tomáš Kočiskỳ, Jonathan Schwarz, Phil Blunsom, Chris Dyer, Karl Moritz Hermann, Gáabor Melis, and Edward Grefenstette. 2018. The narrativeqa reading comprehension challenge. Transactions of the Association of Computational Linguistics, 6:317-328. 
John Lee and Dag Haug. 2010. Porting an ancient greek and latin treebank. In International Conference on Language Resources and Evaluation.

Jiaming Luo, Yuan Cao, and Regina Barzilay. 2019. Neural decipherment via minimum-cost flow: from ugaritic to linear b. arXiv preprint arXiv:1906.06718.

Thang Luong, Hieu Pham, and Christopher D Manning. 2015. Effective approaches to attention-based neural machine translation. In Empirical Methods in Natural Language Processing, pages 1412-1421.

Harold Mattingly. 1996. The Athenian empire restored: epigraphic and historical studies. University of Michigan Press.

Tomáš Mikolov, Anoop Deoras, Stefan Kombrink, Lukáš Burget, and Jan Černockỳ. 2011. Empirical evaluation and combination of advanced language modeling techniques. In International Speech Communication Association.

Tomáš Mikolov, Martin Karafiát, Lukáš Burget, Jan Černockỳ, and Sanjeev Khudanpur. 2010. Recurrent neural network based language model. In International Speech Communication Association.

Tomáš Mikolov, Ilya Sutskever, Anoop Deoras, HaiSon Le, Stefan Kombrink, and Jan Cernocky. 2012. Subword language modeling with neural networks. Preprint, 8.

Yasumasa Miyamoto and Kyunghyun Cho. 2016. Gated word-character recurrent language model. In Empirical Methods in Natural Language Processing, pages 1992-1997.

Émilie Pagé-Perron, Maria Sukhareva, Ilya Khait, and Christian Chiarcos. 2017. Machine translation and automated analysis of the sumerian language. In Association for Computational Linguistics.

Satish Palaniappan and Ronojoy Adhikari. 2017. Deep learning the indus script. arXiv preprint arXiv:1702.00523.

Michail Panagopoulos, Constantin Papaodysseus, Panayiotis Rousopoulos, Dimitra Dafi, and Stephen Tracy. 2009. Automatic writer identification of ancient greek inscriptions. Transactions on pattern analysis and machine intelligence, 31(8):14041414.

Rajesh PN Rao, Nisha Yadav, Mayank N Vahia, Hrishikesh Joglekar, R Adhikari, and Iravatham Mahadevan. 2009a. Entropic evidence for linguistic structure in the indus script. Science, 324(5931):1165-1165.

Rajesh PN Rao, Nisha Yadav, Mayank N Vahia, Hrishikesh Joglekar, R Adhikari, and Iravatham Mahadevan. 2009b. A markov model of the indus script. Proceedings of the National Academy of Sciences, 106(33):13685-13690.
Rajesh PN Rao, Nisha Yadav, Mayank N Vahia, Hrishikesh Joglekar, Ronojoy Adhikari, and Iravatham Mahadevan. 2010. Entropy, the indus script, and language. Computational Linguistics, 36(4):795-805.

Peter John Rhodes and Robin Osborne. 2003. Greek historical inscriptions: 404-323 BC. Oxford University Press, USA.

Henriette Roued-Cunliffe. 2010. Towards a decision support system for reading ancient documents. Literary and linguistic computing, 25(4):365-379.

Arie Shaus. 2017. Computer Vision and Machine Learning Methods for Analyzing First Temple Period Inscriptions. Ph.D. thesis, Tel Aviv University.

David Smith, Jeffrey Rydberg-Cox, and Gregory Crane. 2000. The perseus project: A digital library for the humanities. Literary and Linguistic Computing, 15(1):15-25.

A. Soumya and G. Hemantha Kumar. 2014. Classification of ancient epigraphs into different periods using random forests. In International Conference on Signal and Image Processing, pages 171-178. IEEE.

Thendral Suganya and Subramaniam Murugavalli. 2017. Feature selection for an automated ancient tamil script classification system using machine learning techniques. In International Conference on Algorithms, Methodology, Models and Applications in Emerging Technologies, pages 1-6. IEEE.

Ilya Sutskever, James Martens, and Geoffrey E Hinton. 2011. Generating text with recurrent neural networks. In International Conference on Machine Learning, pages 1017-1024.

Ilya Sutskever, Oriol Vinyals, and Quoc V Le. 2014. Sequence to sequence learning with neural networks. In Advances in neural information processing systems, pages 3104-3112.

Melissa Terras and Paul Robertson. 2006. Image to interpretation: an intelligent system to aid historians in reading the Vindolanda texts. Oxford University Press.

The Packard Humanities Institute. 2005. PHI greek inscriptions. https://inscriptions.packhum.org/. Accessed on 2019-04-24.

Stephen V. Tracy and Constantin Papaodysseus. 2009. The study of hands on greek inscriptions: The need for a digital approach. American Journal of Archaeology, 113(1):99-102.

Alessandro Vatri and Barbara McGillivray. 2018. The diorisis ancient greek corpus. Research Data Journal for the Humanities and Social Sciences.

Arthur Woodhead. 1967. The study of Greek inscriptions, volume 424. Cambridge University Press Archive. 
Qizhe Xie, Guokun Lai, Zihang Dai, and Eduard Hovy. 2018. Large-scale cloze test dataset created by teachers. In Empirical Methods in Natural Language Processing, pages 2344-2356.

Nisha Yadav, Hrishikesh Joglekar, Rajesh PN Rao, Mayank N Vahia, Ronojoy Adhikari, and Iravatham Mahadevan. 2010. Statistical analysis of the indus script using n-grams. PLoS One, 5(3):e9506.

Wojciech Zaremba, Ilya Sutskever, and Oriol Vinyals. 2015. Recurrent neural network regularization. In International Conference on Learning Representations.

Zhuosheng Zhang, Yafang Huang, Pengfei Zhu, and Hai Zhao. 2018. Effective character-augmented word embedding for machine reading comprehension. In CCF International Conference on Natural Language Processing and Chinese Computing, pages 27-39. Springer. 